\title{
Deep learning Convolutional Neural Network for Unconstrained License Plate Recognition
}

\author{
Yee Yong Pang ${ }^{1, *}$, Chee Hau $\mathrm{Ong}^{2 *}$, and Hiew Moi $\mathrm{Sim}^{1}$ \\ ${ }^{1}$ Department of Computer Science, Faculty of Engineering \& Information Technology, Southern University College, Jalan Selatan \\ Utama, Off Jalan Skudai, 81300 Skudai, Johor, Malaysia \\ ${ }^{2}$ Computer Centre, Southern University College, Jalan Selatan Utama, Off Jalan Skudai, 81300 Skudai, Johor, Malaysia
}

\begin{abstract}
The evolve of neural networks algorithm into deep learning convolutional neural networks seems like the next generation for object detection. This algorithm works has a significantly better accuracy and did not tied to any particular aspect ratio. License plate and traffic signs detection and recognition have a number of different applications relevant for transportation systems, such as traffic monitoring, detection of stolen vehicles, driver navigation support or any statistical research. An exponential increase in number of vehicles necessitates the use of automated systems to maintain vehicle information. The information is highly required for both management of traffic as well as reduction of crime. Number plate recognition is an effective way for automatic vehicle identification. A number of methods have been proposed, but only for particular cases and working under constraints (e.g. known text direction or high resolution). Deep learning convolutional neural networks work well especially in handles occlusion/rotation better, therefore we believe this approach is able to provide a better solution to the unconstrained license plate recognition problem.
\end{abstract}

\section{Introduction}

License plate recognition is a well-known image processing technology, which may collect license plate information after capture and analyze the target transportation's plate. In this era license plate recognition technology normally will be used in security platform such as finding stolen cars, traffic monitoring, or other function like Toll/Parking fee payment, navigation support and so on. [1]

LPRS (License Plate Recognition System) may accurately analyze the license plate with nearly $100 \%$ success rate with constrained license plate, but unconstrained license plate will affect the analytic result such as broken license plate, low resolution, bad weather or low light environment [2]. Fig. 1 showed the examples of unconstrained licence plate in difference circumstances.

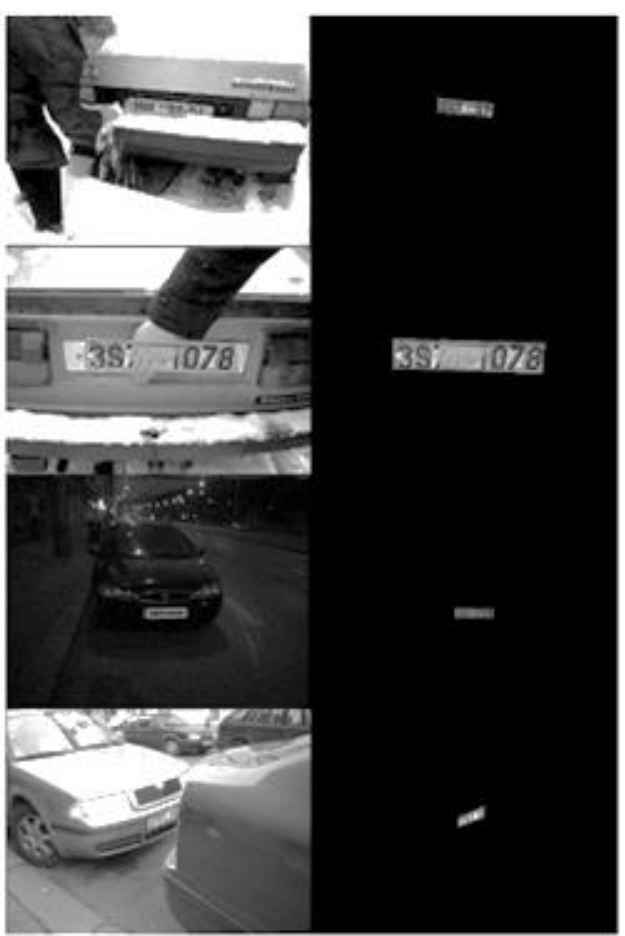

Fig 1. Unconstrained licence plate

To improve this kind of analytics, Convolutional Neural Network will be used in this article. Convolutional neural

Corresponding author: yypang@sc.edu.my, chong@sc.edu.my 
network model as well as neocognitrons known as one of biologically inspired models, have been used for pattern recognition tasks such as face recognition and handwritten numeral recognition [3].

License plate recognize nowadays having high accuracy under constrained dataset, but not all technique may fully recognize the unconstrained license plate, it may affect the result of statistic and wrong recognize the license plate which may decrease the effectiveness of surveillance system. Deep learning convolutional neural networks work well especially in handles occlusion/rotation better, therefore we believe this approach is able to provide a better solution to the unconstrained license plate recognition problem.

\section{Challenge and Objectives}

There are few challenges need to face and overcome it in case to improve the accuracy of detection. Basically there are five steps of process to analyses an image and detect the character.

First we need to acquire an image from the camera, in unconstrained environments a bad images may affect the next step processing and accuracy. There are few constrained environments like raining day, camera lens irradiated by car head light and long light environment which may get an unclear image and affect the result of analysis. To overcome the problem we need to develop a way to cuts down the light reflections of rain or head light.

In pre-processing process, we need concern about the image quality, shadows, and noises and solve it by image masking and binarization. Since all image source has been tuned to perfect condition we may start to focus on license plate detection.

The license plate position may be different on vehicle, motorcycle and lorry. A right camera position may detect the car plate by using horizontal or vertical segmentation. System will segment character correctly before analyze the character by tilt correction and restore the original scale and shape.

$$
\text { PBO8C06583 }
$$

Fig 2. Extracted License Plate [4]

\section{$P|B| 0|8| C|0| 6|5| 8 \mid 3$}

Fig 3. Segmented Characters [4]

After all things are done, we will use convolutional neural network to analyze the pattern of character and number and developing a way to tuning the accuracy of recognize.
An exponential increase in number of vehicles necessitates the use of automated systems to maintain vehicle information. The information is highly required for both management of traffic as well as reduction of crime.

\section{Finding}

This section will focus on the common process of car plate recognition, and based on different techniques use to increase the adaptability by using a suitable technology or theory.

As an example of recognition process, when a picture taken from the camera and pass the image frame to process, system will try to improve the quality of images by remove shadows and remove noises in the image. Projection scanning will be made to identify the license plate location before extracting the character from the license plate image. The process will be continued by using effective technique to recognize the license plate letter.

There are some bottlenecks in unconstrained environment during pre-processing. Hardware may be one of the major problems in unconstrained environment, regular camera unable to capture clear image due to some of reasons like resolution, filter, fps, infrared support and so on. Fig. 4 showed the difference between the License Plate Capture (LPR) Cameras and regular camera where later recorded a blur image.

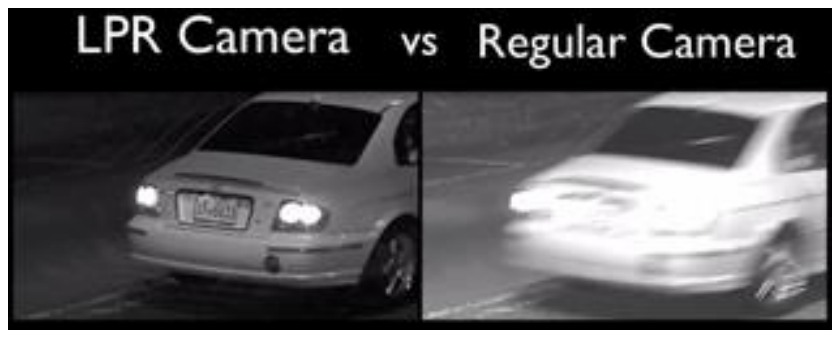

Fig 4. Difference between LPR Camera \& Regular Camera

Besides, the result will also be affected by different weather or different environmental factor like raining day, foggy and smog. 


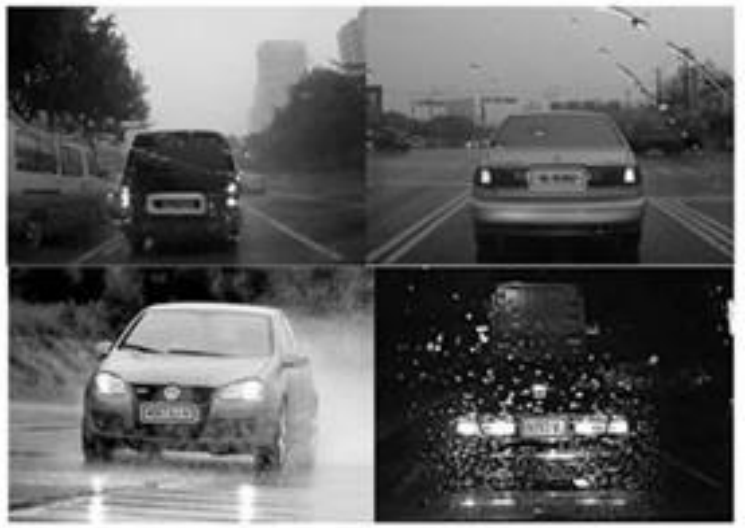

(a) Correctly detected results.

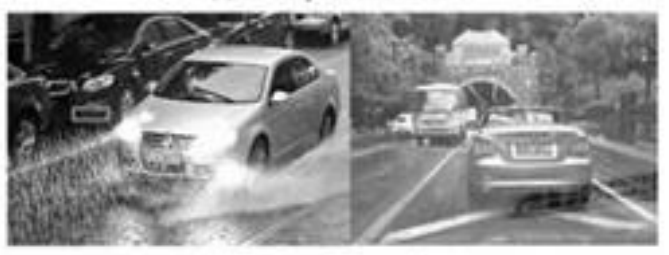

(b) Mis-detected fiemse plates.

Fig 5. Pre-processing bottleneck in unconstrained environment. (Rainy Day). [5]

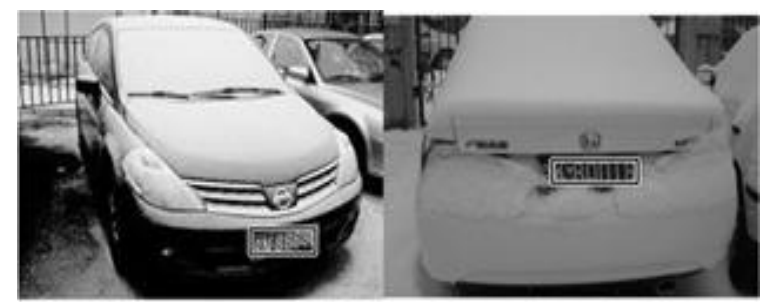

(a) Correctly detected results.

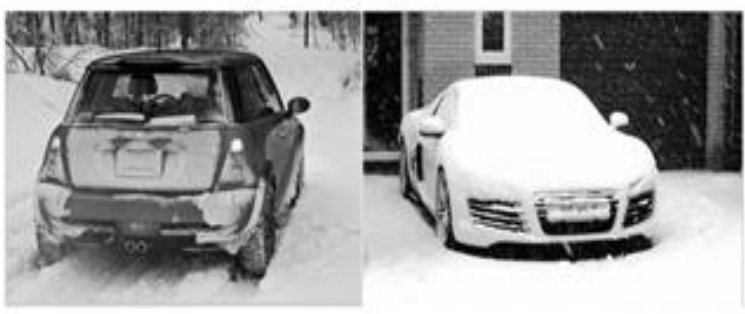

(b) Mis-detected license plates.

Fig 6. Pre-processing bottleneck in unconstrained environment. (Snow Day) [5]

Besides the bottleneck, a high adaptability technique will need to be considered to obtain high success rate of analysis. In the market field, there are different theory and technique used to solve different problem related to this. Search Window Method is one of the methods to analyze the image by horizontal and vertical projections through search window. Edge Analysis is a region with dense vertical edges which segmented as a candidate plate and is known as Region of Interest. A Connected Component Analysis (CCA) is a binary image processing to count the similar labels to distinguish it as a region, and Sliding Concentric Windows (SCW) is a method that used to speed up detection rate of CCA.
Beside this, there is a technique which called Fuzzy Discipline, author framed some rules to explain about the License Plate and gave some membership functions for fuzzy sets - "bright", "dark", "bright and dark sequence", "texture" and "yellowness" to obtain the horizontal and vertical plate positions. Improved Bernsen is used to analysis image with 2 ways, one is detection of white frame and another one is detection of black characters. White frame is detected using CCL technique and it is sensitive to the edges. There're some other similar technique which called Vertical Edge Detection and Hough Transform with Counter.

Beside the License Plate Detection Technique, there are few method which used to recognize the character which called Character Recognition Method. Seven of these are commonly used in industries field which are Artificial Neural Network, SO Neural Network, Probabilistic Neural Network, Support Vector Machine, Template Matching, and Hidden Markov Model.

In our research, we focus on Convolutional Neural Network. This network is inspired by biological processes in which the connectivity pattern between neurons is inspired by the organization of the animal visual cortex. It use a variation of multilayer perceptron designed to require minimal pre-processing. It also known as shift invariant or space invariant artificial neural networks (SIANN).Equations should be centred and should be numbered with the number on the right-hand side.

\section{Conclusions}

Since license plate recognition is a well-known image processing technology, but it seems uncommon product due to high cost of hardware, installation and maintenance fee. This research is going to explore the possibility to achieve low cost device and improve the technology which may suitable using under unconstrained environment. And this research uses Convolutional Neural Network as character recognition method for self-learning purpose to effectively analyze car plate in Malaysia.

\section{References}

1. J. Matas, K. Zimmermann, Unconstrained licence plate and text localization and recognition, Proceedings in 2005 IEEE Intelligent Transportation Systems, pp. 225230, (2005)

2. H. Karwal, A. Girdhar, Vehicle Number Plate Detection System for Indian Vehicles, 2015 IEEE International Conference on Computational Intelligence \& Communication Technology, Ghaziabad, pp. 8-12, (2015)

3. M. Matsugu, K. Mori, Y. Mitari, Y. Kaneda, Subject independent facial expression recognition with robust face detection using a convolutional neural network, Neural Networks 16, (2003)

4. S. Kumari, A Robust Method for Vehicle License Plate Recognition based on Harries Algorithm and Artificial Neural Network, International Journal of Computer Applications 148 - No. 4, (2016) 
5. Y-N. Chen, C-C. Han, G-F. Ho, K-C. Fan, Facial/license plate detection using a two-level cascade classifier and a single convolutional feature map, International Journal of Advanced Robotic Systems, (2015) 